PHYSICAL REVIEW D 89, 061501(R) (2014)

\title{
Stability of nonspinning effective-one-body model in approximating two-body dynamics and gravitational-wave emission
}

\author{
Yi Pan, ${ }^{1}$ Alessandra Buonanno, ${ }^{1}$ Andrea Taracchini, ${ }^{1}$ Michael Boyle, ${ }^{2}$ Lawrence E. Kidder, ${ }^{2}$ Abdul H. Mroué, ${ }^{3}$ \\ Harald P. Pfeiffer, ${ }^{3,4}$ Mark A. Scheel, ${ }^{5}$ Béla Szilágyi, ${ }^{5}$ and Anil Zenginoglu ${ }^{5}$ \\ ${ }^{1}$ Department of Physics, Maryland Center for Fundamental Physics \& Joint Space-Science Institute, \\ University of Maryland, College Park, Maryland 20742, USA \\ ${ }^{2}$ Center for Radiophysics and Space Research, Cornell University, Ithaca, New York 14853, USA \\ ${ }^{3}$ Canadian Institute for Theoretical Astrophysics, University of Toronto, \\ 60 St. George Street, Toronto, Ontario M5S 3H8, Canada \\ ${ }^{4}$ Canadian Institute for Advanced Research, 180 Dundas Street West, Toronto, Ontario M5G 1Z8, Canada \\ ${ }^{5}$ Theoretical Astrophysics 350-17, California Institute of Technology, Pasadena, California 91125, USA
} (Received 11 November 2013; published 5 March 2014)

\begin{abstract}
The detection of gravitational waves and the extraction of physical information from them requires the prediction of accurate waveforms to be used in template banks. For that purpose, the accuracy of effectiveone-body (EOB) waveforms has been improved over the last years by calibrating them to numerical-relativity (NR) waveforms. So far, the calibration has employed a handful of NR waveforms with a total length of $~ 30$ cycles, the length being limited by the computational cost of NR simulations. Here, we address the outstanding problem of the stability of the EOB calibration with respect to the length of NR waveforms. Performing calibration studies against NR waveforms of nonspinning black-hole binaries with mass ratios 1 , $1.5,5$ and 8 , and with a total length of $\sim 60$ cycles, we find that EOB waveforms calibrated against either 30 or 60 cycles will be indistinguishable by the advanced detectors Laser Interferometric Gravitational-wave Observatory (LIGO) and Virgo when the signal-to-noise ratio (SNR) is below 110. When extrapolating to a very large number of cycles, using very conservative assumptions, we can conclude that state-of-the-art nonspinning EOB waveforms of any length are sufficiently accurate for parameter estimation with advanced detectors when the SNR is below 20, the mass ratio is below 5 and the total mass is above $20 M_{\odot}$. The results are not conclusive for the entire parameter space because of current NR errors.
\end{abstract}

DOI: 10.1103/PhysRevD.89.061501

\section{INTRODUCTION}

Coalescing compact-object binaries are among the most promising gravitational-wave (GW) sources for ground-based interferometric detectors such as Laser Interferometric Gravitational-wave Observatory (LIGO), Virgo, and The Kamioka Gravitational Wave Detector (KAGRA) [1-3]. Accurate waveform models are crucial for detecting the signals and measuring the physical parameters of the sources. By solving the Einstein equations numerically [4], it is possible to produce accurate waveforms for the very late inspiral, merger and ringdown stages of the coalescence process. However, the length of numerical-relativity (NR) simulations is limited by their high computational cost, and today it is unrealistic to generate sufficiently many NR waveforms long enough to be used directly in GW searches. The post-Newtonian (PN) formalism [5] is a slow-motion, weak field approximation to the Einstein field equations that provides reliable low-frequency inspiral waveforms. However, the PN approach becomes increasingly inaccurate close to merger [6]. Several studies [7-9] showed that there is a substantial gap between the frequency $f_{\mathrm{PN}}$ where PN waveforms cease being accurate and the frequency $f_{\mathrm{NR}}$ where NR simulations start being available. The width of the frequency gap $f_{\mathrm{NR}}-f_{\mathrm{PN}}$ depends on source parameters,
PACS numbers: 04.25.D-, 04.25.dg, 04.25.Nx, 04.30.-w

and it is generally believed to increase rapidly with increasing mass ratios and spin magnitudes. Much longer NR simulations can reduce $f_{\mathrm{NR}}$ while knowledge of higher-order PN terms in the two-body dynamics and radiation-reaction force can increase $f_{\mathrm{PN}}$ [9], but it is extremely challenging to achieve those goals. An accurate description of the waveform in the frequency gap is thus an outstanding and pressing problem of GW source modeling, especially because advanced detectors will be operational in a few years.

The effective-one-body (EOB) formalism [10] is a successful approach that provides a complete description of the coalescence of compact-object binaries. It uses the $\mathrm{PN}$-expanded results in a resummed form and incorporates results of black-hole perturbation theory to produce waveforms for the inspiral, merger and ringdown stages. By construction, the EOB model reduces to the PN approximation at low frequency, while in the strong-field regime it models the merger and ringdown signals using physically motivated guesses and insights from perturbation theory. Following the breakthrough in merger simulations in NR [11], the EOB model has been improved by calibrating it to progressively more accurate and longer NR simulations, also spanning larger regions of the parameter space [12-16]. Considering the success in calibrating NR 
waveforms, we expect that the EOB model will be able to interpolate/extrapolate NR waveforms over the entire source parameter space. However, it is not yet clear whether the EOB calibration is stable under variations of the length of the NR waveforms that are used to calibrate the model, and whether EOB waveforms of lengths larger than the one used for calibration can safely be used to detect GW signals and extract physical parameters with advanced detectors.

In this paper, we focus on the low-frequency, inspiral performance of the EOB model and assume, based on previous calibrations, that calibrated EOB merger and ringdown waveforms can be made indistinguishable from the NR ones [17]. The EOB adjustable parameters that are used to calibrate the model not only improve EOB waveforms at high frequency, so that they match NR waveforms very accurately above $f_{\mathrm{NR}}$, but they also introduce deviations from known PN results in the frequency gap $f_{\mathrm{PN}}-f_{\mathrm{NR}}$. Below $f_{\mathrm{PN}}$, all PN-waveform families and the EOB waveforms agree with each other. The goal of this paper is to understand the accuracy of the EOB waveforms in the frequency gap, addressing the following questions: Is the EOB calibration stable with respect to the length of NR waveforms (i.e., with respect to varying $f_{\mathrm{NR}}$ )? If the calibration is stable when using the current length of NR simulations, for which we still have $f_{\mathrm{NR}} \gg f_{\mathrm{PN}}$, can we conclude that the calibrated EOB waveforms will be indistinguishable from the exact ones for all frequencies below $f_{\mathrm{NR}}$ ?

\section{A. Calibrating the effective-one-body model}

We calibrate the EOB model against four nonspinning binary black-hole waveforms with mass ratios $q=1,1.5,5$ and 8 . The $q=1$ simulation was first presented in [9], and all four simulations are presented in [18]. Table I lists the total number of GW cycles of the NR waveforms up to merger, including the junk radiation, and the maximum number of cycles $N_{\max }$ that we use when calibrating the EOB model (i.e., after removing the junk radiation). We decompose the EOB waveforms in 2 spin-weighted spherical-harmonic modes $(\ell, m)$. Previous studies [12] have shown that during the inspiral stage the frequency of all modes is well approximated by the $m$ multiple of the orbital frequency. Therefore, for simplicity, we consider only the dominant $(\ell=2, m=2)$ mode. We expect that the results of our study hold to a considerable extent for the other modes since phase evolution of every mode is synchronized

TABLE I. Total number of GW cycles $N_{\text {sim }}$ of NR simulations (including junk radiation) up to merger and maximum number of cycles $N_{\max }$ used for EOB-model calibration, i.e., without junk radiation.

\begin{tabular}{lcccc}
\hline \hline$q$ & 1 & 1.5 & 5 & 8 \\
\hline$N_{\text {sim }}$ & 65 & 66 & 58 & 52 \\
$N_{\max }$ & 60 & 60 & 55 & 50 \\
\hline \hline
\end{tabular}

with the orbital phase. However, since higher-order modes give smaller contributions and have larger NR errors, it will be more challenging to extend the current study to these other modes.

The EOB inspiral-plunge dynamics for quasicircular orbits is described by a set of Hamilton equations that include a dissipative force proportional to the rate of loss of the orbital energy. One then introduces adjustable parameters, i.e., unknown, higher-order PN terms, to improve both conservative and dissipative parts of the dynamics. To match the EOB to the NR waveforms within the NR error, only a few adjustable parameters are needed and their choice is not unique. In the nonspinning limit, the EOB model depends only on two (or even one [19]) adjustable parameters $A^{(i)}, i=1,2$. We follow the parametrization of Ref. [13], where two adjustable parameters were used in the nonspinning sector. The EOB inspiral waveform of mass ratio $q$ is therefore determined by the pair $\left\{A^{(1)}, A^{(2)}\right\}$, where these coefficients depend on the mass ratio $q$. We calibrate the EOB model by mapping the phase difference between EOB and NR waveforms in the $A^{(1)}-A^{(2)}$ parameter space, taking into account NR errors in the simulations.

In our calibration procedure, we measure the phase difference at the end of inspiral, after aligning the EOB and NR waveforms at low frequency by shifting the EOB waveform in time and phase. We determine the time and phase shifts $\bar{t}_{0}$ and $\bar{\phi}_{0}$ by minimizing the square of the difference between the GW phases of the NR and EOB waveforms,

$$
\int_{t_{1}}^{t_{2}}\left[\phi_{22}^{\mathrm{EOB}}\left(t+t_{0}\right)+\phi_{0}-\phi_{22}^{\mathrm{NR}}(t)\right]^{2} d t
$$

with respect to $t_{0}$ and $\phi_{0}$. The phase difference at a given time is given by

$$
\Delta \phi(t)=\phi_{22}^{\mathrm{EOB}}\left(t+\bar{t}_{0}\right)+\bar{\phi}_{0}-\phi_{22}^{\mathrm{NR}}(t),
$$

where $\bar{t}_{0}$ and $\bar{\phi}_{0}$ are the alignment parameters that minimize Eq. (1). The global phase difference over a time window $\left(t_{1}, t_{3}\right)$ is defined as

$$
\Delta \phi_{g}=\max _{t \in\left(t_{1}, t_{3}\right)}|\Delta \phi(t)| .
$$

We set $t_{3}$ to the time of merger, i.e., to the time at which $\left|h_{22}^{\mathrm{EOB}}\right|$ reaches its maximum. Here, we are interested in the inspiral performance; thus we ignore the phase difference beyond the time of merger, which is affected by the procedure of building the merger-ringdown waveform. Because of NR errors in $\phi_{22}^{\mathrm{NR}}$, the time shift $t_{0}$ and the global phase difference $\Delta \phi_{g}$ are rather sensitive to the choice of the time window $\left(t_{1}, t_{2}\right)$. To alleviate the effect of NR errors, we choose $\left(t_{1}, t_{2}\right)$, following the prescription of Ref. [13]. We also repeat the alignment using four different choices of $\left(t_{1}, t_{2}\right)$ to estimate the uncertainty of $\Delta \phi_{g}$ due to 

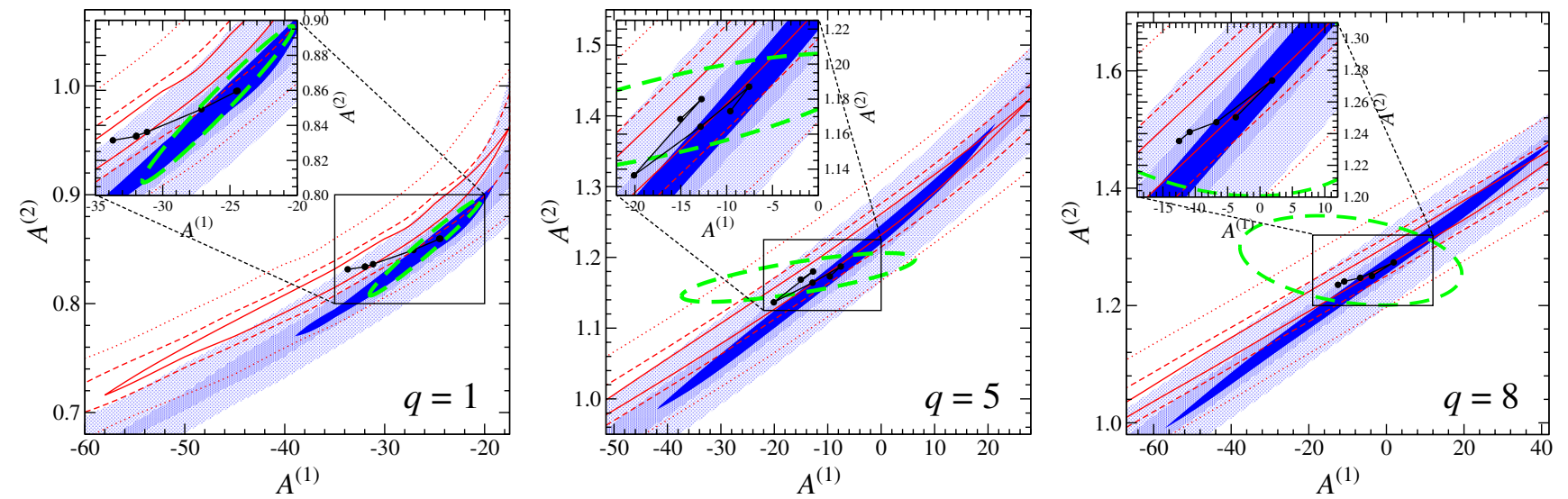

FIG. 1 (color online). Contours of the global phase difference $\Delta \phi_{g}$ between nonspinning NR waveforms of $N$ GW cycles and EOB waveforms with adjustable parameters $\left\{A^{(1)}, A^{(2)}\right\}$. The three panels show results for mass ratios $q=1,5$ and 8 (from left to right). The shaded regions, from inside out, are $0.1,0.2$ and 0.5 radian contours for comparisons with $N_{\max }$ cycles of NR waveforms. The solid, dashed and dotted lines are the same contours for comparisons with 30 cycles of NR waveforms. The connected black dots are the calibrated points $\left\{\bar{A}_{N}^{(1)}(q), \bar{A}_{N}^{(2)}(q)\right\}$ for $N$ values changing from 30 to $N_{\max }$. The inset zooms around these points. The NR error box of the calibrated point $\left\{\bar{A}_{N_{\max }}^{(1)}(q), \bar{A}_{N_{\max }}^{(2)}(q)\right\}$ is shown with the dashed ellipse.

NR errors. To calibrate the EOB model, we find those parameters $\left\{\bar{A}_{N}^{(1)}(q), \bar{A}_{N}^{(2)}(q)\right\}$ that minimize $\Delta \phi_{g}$. The subscript $N$ indicates that calibration was performed using the last $N \mathrm{GW}$ cycles, i.e. $t_{1}$ corresponds to a time $N$ cycles before merger, and $t_{3}$ is at merger. When building a calibrated EOB model [13], we fit the calibrated points $\left\{\bar{A}_{N}^{(1)}(q), \bar{A}_{N}^{(2)}(q)\right\}$ to a smooth function in $q$. However, since the fits' residuals are typically smaller than the NR errors, we use the calibrated points instead of the fitted functions. We then increase $N$ from 30 to $N_{\max }$ with a step size of 5 and determine how the point $\left\{\bar{A}_{N}^{(1)}(q), \bar{A}_{N}^{(2)}(q)\right\}$ moves in the parameter space. Besides systematic errors in the EOB model, the calibration point can also change because of the NR errors.

The NR errors affect $\left\{\bar{A}_{N}^{(1)}(q), \bar{A}_{N}^{(2)}(q)\right\}$ in two ways. The oscillatory phase errors at low frequency (due to residual eccentricity) introduce uncertainties in the alignment procedure, while the secular phase errors introduce uncertainties directly in the global phase difference $\Delta \phi_{g}$. To estimate the impact of those NR errors on $\left\{\bar{A}_{N}^{(1)}(q), \bar{A}_{N}^{(2)}(q)\right\}$, we calculate those calibrated points using four different choices of the alignment time window $\left(t_{1}, t_{2}\right)$ and three numerical waveforms: (i) the high resolution, extrapolated to infinity with polynomial degree 3 , (ii) the high resolution, extrapolated to infinity with polynomial degree 4, and (iii) the medium resolution, extrapolated to infinity with polynomial degree 3 . The differences between these numerical waveforms represent the typical truncation and extrapolation errors. Since we are only interested in the position (mean) and spread (variance) of $\left\{\bar{A}_{N}^{(1)}(q), \bar{A}_{N}^{(2)}(q)\right\}$, we do not investigate higher central moments and assume, for simplicity, a bivariate normal distribution of $\left\{\bar{A}_{N}^{(1)}(q), \bar{A}_{N}^{(2)}(q)\right\}$. We use the 12 data points to calculate the maximum likelihood estimators of their mean and variance.

We summarize our results in Fig. 1 for $q=1,5$, and 8 and omit the $q=1.5$ case because it is very similar to the $q=1$ case. When $N$ increases from 30 to $N_{\max }$, the volume enclosed by the $\Delta \phi_{g}$ contours decreases gradually, reflecting tighter constraints from the calibration against longer NR simulations. Somewhat unexpectedly, the contours also shift and rotate smoothly, indicating a possible systematic change of the calibrated EOB model. For clarity, we show in Fig. 1 only the contours of $N=30$ and $N_{\max }$ calibrations. In the inset of each panel, we zoom in around the calibrated points $\left\{\bar{A}_{N}^{(1)}(q), \bar{A}_{N}^{(2)}(q)\right\}$ to show their path when $N$ changes from 30 to $N_{\max }$. We also show the NR error box of $\left\{\bar{A}_{N_{\max }}^{(1)}(q), \bar{A}_{N_{\max }}^{(2)}(q)\right\}$, which is the symmetric $95 \%$ quantile of the estimated bivariate normal distributions. In the $q=1$ case, the systematic drift of $\left\{\bar{A}_{N}^{(1)}(1), \bar{A}_{N}^{(2)}(1)\right\}$ with increasing $N$ is not fully accounted for by the NR errors. Of course, it is in principle possible to improve the accuracy of the EOB model by calibrating it to the $N_{\max }$-cycle numerical waveforms. However, since the systematic differences between $\left\{\bar{A}_{N}^{(1)}(1), \bar{A}_{N}^{(2)}(1)\right\}$ are not much larger than the NR error boxes, the NR waveforms have to be as accurate as the $q=1$ waveforms employed in this paper to bring new information to the EOB calibration. For instance, the calibrated point $\left\{\bar{A}_{30}^{(1)}(1), \bar{A}_{30}^{(2)}(1)\right\}$ sits on the 0.5 -radian contour of $\Delta \phi_{g}$ obtained from the $N=60$ calibration. That is to say, if a $q=1,60$-cycle NR waveform and a 60-cycle EOB waveform generated by a model calibrated to a 30-cycle NR waveform, such as the EOB model in Ref. [13], are aligned, their accumulated phase difference at merger is only $\sim 0.5$ radians. Any NR phase error at a merger larger than that, accumulated over 60 
cycles, would not improve the low-frequency accuracy of the EOB model. In fact, the $q=5$ and $q=8$ NR waveforms, despite being rather long and accurate, do not provide new information to the EOB calibration. Truncation errors of these simulations dominate over other numerical errors and EOB modeling errors. More accurate NR simulations of large $q$ are therefore needed to further improve the low-frequency accuracy of the EOB model.

\section{B. Stability of the EOB calibration}

Although the differences among $\left\{\bar{A}_{N}^{(1)}(1), \bar{A}_{N}^{(2)}(1)\right\}$ waveforms can be distinguished by the global phase difference $\Delta \phi_{g}$, which is a highly sensitive quantity, it is not clear whether they can be distinguished by interferometric advanced detectors, such as LIGO. Using the zerodetuned high-power advanced LIGO noise curve [1] and a total mass for the black-hole binary of $20 M_{\odot}$, we quantify the data-analysis consequence of the differences between $\left\{\bar{A}_{N}^{(1)}(q), \bar{A}_{N}^{(2)}(q)\right\}$. Our study follows the procedure of Ref. [9] and our results can be compared directly with those of Ref. [9].

First, we employ the quantity $\|d h\| /\|h\|$ [20] to measure the difference between waveforms $h_{1}$ and $h_{2}$, where $d h \equiv h_{1}-h_{2}, h=h_{1}$. The norm is defined through the inner-product $\left\langle h_{1}, h_{2}\right\rangle \equiv 4 \operatorname{Re} \int_{0}^{\infty}\left(\tilde{h}_{1}(f) \tilde{h}_{2}^{*}(f)\right) / S_{n}(f) d f$, where $S_{n}(f)$ is the noise spectral density.

When we minimize over time and phase of coalescence, as well as physical parameters, $\|d h\| /\|h\|$ measures the relative loss of signal-to-noise ratio (SNR). When we minimize over only the time and phase of coalescence, $\|d h\| /\|h\|$ measures the bias in measuring source parameters due to modeling errors. The bias is less than statistical errors when $\|d h\| /\|h\|<1 / \rho_{\text {eff }}$, where the effective SNR $\rho_{\text {eff }}=1 / \varepsilon \sqrt{n_{D}} \rho$ is proportional to the single-detector SNR $\rho$ with a coefficient given by the number of detectors $n_{D}$ and a safe factor $1 / \varepsilon$ [7] of order unity. Satisfying this condition means that the detector cannot distinguish $h_{1}$ and $h_{2}$. Either is an accurate enough template to measure the source parameters of the other. (We emphasize that the criterion of indistinguishability proposed in Ref. [20], i.e., $\|d h\|<1$, is a sufficient but not necessary criterion, and it has been shown to be highly restrictive [17].)

In order to calculate $\|d h\| /\|h\|$, we need to complete the EOB inspiral waveforms $\left\{\bar{A}_{N_{\max }}^{(1)}(q), \bar{A}_{N_{\max }}^{(2)}(q)\right\}$ with merger and ringdown waveforms. Previous studies demonstrated that it is always possible to calibrate the EOB merger and ringdown waveforms to sufficient accuracy once the inspiral waveforms are accurately calibrated $[12,17]$. So, here, we do not include the EOB merger and ringdown waveforms, but simply attach the NR late-inspiral, merger and ringdown waveforms to the EOB inspiral waveforms, starting at the matching frequency $\omega_{m}$; i.e., we construct $\mathrm{EOB}+\mathrm{NR}$ hybrid waveforms. This allows us to directly compare our results with the ones of Ref. [9]. In fact, for

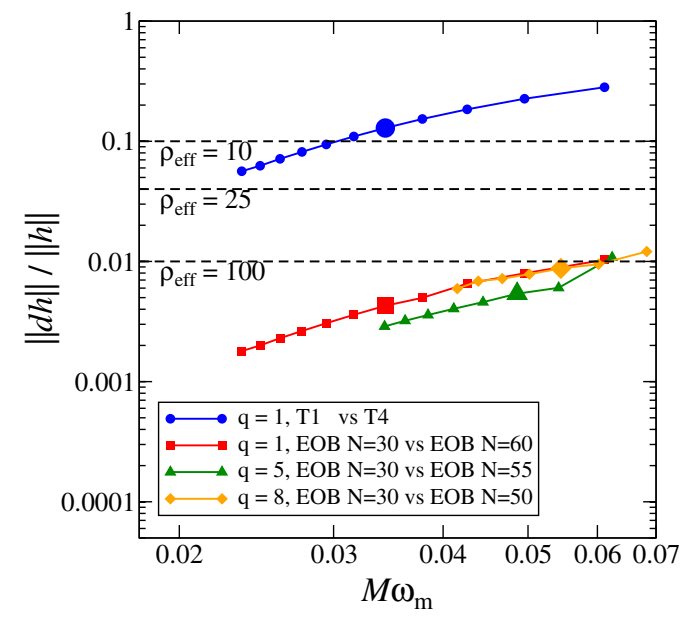

FIG. 2 (color online). $\quad\|d h\| /\|h\|$ minimized over time and the phase of coalescence as a function of the hybrid matching frequency $\omega_{m}$ for $\mathrm{EOB}+\mathrm{NR}$ hybrids where $\mathrm{EOB}$ waveforms are generated with the calibrated points $\left\{\bar{A}_{30}^{(1)}(q), \bar{A}_{30}^{(2)}(q)\right\}$ and $\left\{\bar{A}_{N_{\max }}^{(1)}(q), \bar{A}_{N_{\max }}^{(2)}(q)\right\}$. We also show the same quantity for PN +NR hybrids using TaylorT1 and TaylorT4 approximants. The bigger symbol in each data set marks the matching frequency where the hybrid is built using 30 cycles of NR waveforms. The horizontal lines mark the effective signal-to-noise ratios (SNR)s 10, 25 and 100, below which the difference between waveforms cannot be distinguished by advanced LIGO detectors.

this reason, when building $\mathrm{EOB}+\mathrm{NR}$ waveforms, we also follow the prescription of Ref. [9] on the matching frequency, the time window for alignment and the choice of blending function.

In Fig. 2, we show $\|d h\| /\|h\|$ between $\left\{\bar{A}_{30}^{(1)}(q), \bar{A}_{30}^{(2)}(q)\right\}$ and $\left\{\bar{A}_{N_{\max }}^{(1)}(q), \bar{A}_{N_{\max }}^{(2)}(q)\right\}$ waveforms as a function of the matching frequency. We also include a comparison between PN + NR hybrid waveforms constructed using the TaylorT1 and TaylorT4 approximants [6] as a validation of our code and to compare with Ref. [9]. The difference between $\left\{\bar{A}_{30}^{(1)}(q), \bar{A}_{30}^{(2)}(q)\right\}$ and $\left\{\bar{A}_{N_{\max }}^{(1)}(q), \bar{A}_{N_{\max }}^{(2)}(q)\right\}$ EOB waveforms is more than an order of magnitude smaller than the one obtained using the Taylor-PN approximants. Specifically, when attaching a 30-cycle NR waveform at the end of the EOB inspiral waveform, the difference cannot be distinguished as long as $\rho_{\text {eff }}<110$, which is an unlikely high SNR for advanced detectors [8]. This implies that nonspinning EOB waveforms calibrated to 30 or to $N_{\max }$ cycles of NR waveforms are equivalent when searching for GWs and extracting binary parameters with advanced LIGO detectors. For the EOB model calibrated to 30-cycle NR waveforms, we emphasize that the implication of these results is not just the agreement of its waveform with $N_{\max }$-cycle NR waveforms, but its agreement with the EOB model calibrated to 60-cycle NR simulations, i.e., the stability and convergence of the calibrated EOB model up to 60 cycles. Moreover, this result 


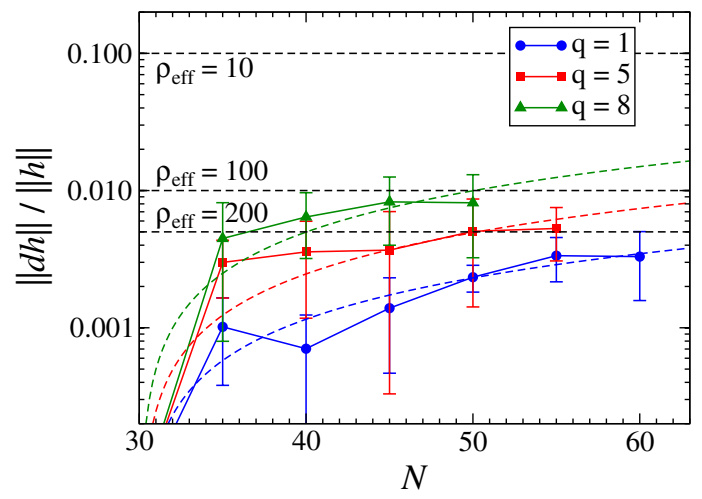

FIG. 3 (color online). $\|d h\| /\|h\|$ between EOB waveforms generated with the calibrated points $\left\{\bar{A}_{30}^{(1)}(q), \bar{A}_{30}^{(2)}(q)\right\}$ and $\left\{\bar{A}_{N}^{(1)}(q), \bar{A}_{N}^{(2)}(q)\right\}$ as a function of the number of NR cycles $N$. We minimize $\|d h\| /\|h\|$ over time and phase of coalescence and use 30 cycles of NR waveforms in the EOB + NR hybrids. The horizontal lines mark the effective SNR 100 and 200, below which the difference between waveforms cannot be distinguished by advanced LIGO detectors.

also demonstrates that calibrated higher-order PN terms (i.e., adjustable parameters) do not have a large effect at low frequency.

Can we extend this conclusion to $N>N_{\max }$ ? In Fig. 3, we show $\|d h\| /\|h\|$ between EOB waveforms computed at the calibrated points $\left\{\bar{A}_{30}^{(1)}(q), \bar{A}_{30}^{(2)}(q)\right\}$ and $\left\{\bar{A}_{N}^{(1)}(q), \bar{A}_{N}^{(2)}(q)\right\}$ as a function of $N$. We see that when $N$ increases from 30 to $N_{\max },\|d h\| /\|h\|$ increases moderately from zero to $<1 \%$ and the increase seems to be slowing down or becoming negative as we approach $N_{\max }$. The oscillations in $\|d h\| /\|h\|$ are consistent with the NR error bars indicated in the plot and estimated using the 12 different $\left\{\bar{A}_{N}^{(1)}(q), \bar{A}_{N}^{(2)}(q)\right\}$ points. If we assume that the very mild increase of $\|d h\| /\|h\|$ is largely explained by NR errors, we might be tempted to conclude that the EOB model has converged beyond $N_{\max }$. However, we must be cautious in extrapolating the results. Nevertheless, it is reasonable to expect that the variation of $\|d h\| /\|h\|$ per unit increase of $N$ eventually becomes a decreasing function of $N$ when $N$ is large enough, and consequently $\|d h\| /\|h\|$ becomes a concave function of $N$. We therefore obtain a conservative estimate of $\|d h\| /\|h\|$ by applying a linear extrapolation of $\|d h\| /\|h\|$ that goes through 0 at $N=30$ and best fits the data points. We find that $\|d h\| /\|h\|<0.05$ until $N=370,235$ and 120 for mass ratios $q=1,5$ and 8 , respectively. That is to say, when $\rho_{\text {eff }} \leq 20$, EOB waveforms calibrated to those numbers of NR cycles cannot be distinguished from EOB waveforms calibrated to 30-cycle NR waveforms. One may hence generate 30-cycle NR simulations to calibrate the EOB model, and use the calibrated model to produce EOB waveforms that are, for data-analysis purposes, identical to NR waveforms of hundreds of cycles.
Finally, we compare these results to the length requirements of NR waveforms set by previous works [7-9] to guarantee the accuracy of PN+NR hybrid waveforms for parameter estimation. Basically, when NR simulations are sufficiently long, their starting frequency $f_{\mathrm{NR}}$ can be reduced to $f_{\mathrm{PN}}$, below which all PN waveform families and PN-based EOB models are consistent. Direct estimates of the number of NR cycles before merger required for accurate hybrid waveforms were made in Ref. [7] (see the table in Fig. 4 of Ref. [7]). When $\rho_{\text {eff }} \leq 20$, for advanced LIGO detectors, the number of GW cycles required for $q=1,4$ and 10 nonspinning NR simulations is 12,190 and 1268 , respectively. Combining those results with ours, we conclude that when $\rho_{\text {eff }} \leq 20$ and $q \leq 5$ the nonspinning EOB waveforms of any length are sufficiently accurate for parameter estimation with advanced LIGO detectors. Note again that these EOB waveforms are generated by the EOB model calibrated to only 30-cycle NR simulations.

\section{CONCLUSIONS}

We found that the EOB-model calibration against NR simulations is stable with respect to the length of NR simulations. In the nonspinning limit with mass ratio $q \leq 8$, the difference between EOB waveforms calibrated against 30-cycle and 260 -cycle NR simulations cannot be distinguished by advanced LIGO detectors when $\rho_{\text {eff }}<110$. Extrapolating our results to a larger number of cycles, making rather conservative assumptions, which use the overstrict criterion from Ref. [20], we estimated that the nonspinning EOB model calibrated to existing NR simulations is sufficiently accurate for advancedLIGO parameter estimation when $\rho_{\text {eff }}<20, q<5$ and $M \geq 20 M_{\odot}$. Moreover, since EOB waveforms overcome the frequency gap, they can completely replace PN + NR hybrid waveforms $[8,9]$. Extending this conclusion to larger $\rho_{\text {eff }}$ or $q$ requires longer and more accurate NR simulations. We plan in the near future to extend this kind of study to the spinning EOB model [19]. We expect that in the presence of spins, we might need longer and more accurate NR simulations, especially in the extremal-spin limit, but the length can be much less than those suggested by previous studies that aimed at reducing $f_{\mathrm{NR}}$ to $f_{\mathrm{PN}}$.

\section{ACKNOWLEDGMENTS}

A. B., Y. P. and A. T. acknowledge partial support from NSF Grants No. PHY-0903631 and No. PHY-1208881, and NASA Grant No. NNX09AI81 G. A. T. also acknowledges support from the Maryland Center for Fundamental Physics. A. M. and H.P acknowledge support from NSERC of Canada, from the Canada Research Chairs Program, and from the Canadian Institute for Advanced Research. M. S. and B. S. gratefully acknowledge support from the Sherman Fairchild Foundation and from NSF 
Grants No. PHY-106881 and No. PHY-1005655 at Caltech. M. B. and L. K. gratefully acknowledge support from the Sherman Fairchild Foundation and from NSF Grants No. PHY-1306125 and No. PHY-1005426 at Cornell. The numerical relativity simulations were performed at the GPC supercomputer at the SciNet HPC Consortium. SciNet is funded by the Canada Foundation for Innovation
(CFI) under the auspices of Compute Canada, the Government of Ontario, Ontario Research FundResearch Excellence, and the University of Toronto. Further computations were performed on the Caltech compute cluster Zwicky, which was funded by the Sherman Fairchild Foundation and the NSF MRI-R2 Grant No. PHY-0960291.
[1] D. Shoemaker (LIGO Collaboration), Report No. T0900288-v3 [https://dcc.ligo.org/cgi-bin/DocDB/ ShowDocument?docid=T0900288.

[2] Virgo Collaboration, Report No. vIR027A09 [https://tds .ego-gw.it/itf/tds/file.php?callFile=VIR-0027A-09.pdf].

[3] K. Somiya and KAGRA Collaboration, Classical Quantum Gravity 29, 124007 (2012).

[4] J. Centrella, J. G. Baker, B. J. Kelly, and J. R. van Meter, Rev. Mod. Phys. 82, 3069 (2010).

[5] L. Blanchet, Living Rev. Relativity 9, 4 (2006).

[6] A. Buonanno, B. Iyer, E. Ochsner, Y. Pan, and B. Sathyaprakash, Phys. Rev. D 80, 084043 (2009).

[7] T. Damour, A. Nagar, and M. Trias, Phys. Rev. D 83, 024006 (2011).

[8] M. Boyle, Phys. Rev. D 84064013 (2011); F. Ohme, M. Hannam, and S. Husa, Phys. Rev. D 84, 064029 (2011); I. MacDonald, S. Nissanke, and H. P. Pfeiffer, Classical Quantum Gravity 28, 134002 (2011).

[9] I. MacDonald, A. H. Mroué, H. P. Pfeiffer, M. Boyle, L. E. Kidder, M. A. Scheel, B. Szilágyi, and N. W. Taylor, Phys. Rev. D 87, 024009 (2013).

[10] A. Buonanno and T. Damour, Phys. Rev. D 59, 084006 (1999); A. Buonanno and T. Damour, Phys. Rev. D 62, 064015 (2000).
[11] F. Pretorius, Phys. Rev. Lett. 95, 121101 (2005); M. Campanelli, C. Lousto, and Y. Zlochower, Phys. Rev. D 73, 061501 (2006); J. G. Baker, J. Centrella, D.-I. Choi, M. Koppitz, and J. van Meter, Phys. Rev. Lett. 96, 111102 (2006).

[12] Y. Pan, A. Buonanno, M. Boyle, L. T. Buchman, L. E. Kidder, H. P. Pfeiffer, and M. A. Scheel, Phys. Rev. D 84, 124052 (2011).

[13] A. Taracchini, Y. Pan, A. Buonanno, E. Barausse, M. Boyle, T. Chu, G. Lovelace, H. P. Pfeiffer, and M. A. Scheel, Phys. Rev. D 86, 024011 (2012).

[14] T. Damour, A. Nagar, S. Bernuzzi, Phys. Rev. D 87, 084035 (2013).

[15] Y. Pan et al., arXiv:1307.6232 [Phys. Rev. D (to be published)].

[16] I. Hinder et al. (NRAR Collaboration), Classical Quantum Gravity 31, 025012 (2014).

[17] T. B. Littenberg, J. G. Baker, A. Buonanno, and B. J. Kelly, Phys. Rev. D 87, 104003 (2013).

[18] A.H. Mroue et al., Phys. Rev. Lett. 111, 241104 (2013).

[19] A. Taracchini et al. (work in progress).

[20] L. Lindblom, Phys. Rev. D 80, 064019 (2009). 